\title{
MATRIX SPECTRAL PROBLEM WITH MULTIPLE-ORDER JUMPS AND POLES
}

\author{
ZHUHAN JIANG ${ }^{1}$
}

(Received 30 August 1993)

\begin{abstract}
The inverse spectral method for a general $N \times N$ spectral problem for solving nonlinear evolution equations in one spacial and one temporal dimension is extended to include multi-boundary jumps and high-order poles and their explicit representations. It therefore provides a formalism to generate soliton solutions that correspond to higher-order poles of the spectral data.
\end{abstract}

\section{Introduction}

Spectral problems in one space dimension have already been extensively studied, see for example $[2,3,6,7]$; the main structure has a solid foundation. A general case of the form $[7,8]$

$$
\psi_{x}=\lambda(k) \psi+u(x, k) \psi,
$$

where $\lambda=\operatorname{diag}\left(\lambda_{1}, \ldots, \lambda_{N}\right)$ and $u$ are $N \times N$ matrices, $u \rightarrow 0$ for $|x| \rightarrow \infty$ and $\lambda(k)$ is analytic in the complex plane $\mathbb{C}$, was studied under essentially a restriction of simple boundary jumps and simple poles on the spectral data. In fact, the apparently more general form in $[7,8]$ is basically reducible to (1). For simplicity we assume $\lambda_{l} \not \equiv \lambda_{m} \forall l \neq m$. Recently high-order poles [12] were introduced algebraically to the ZS-AKNS spectral equation [2] without imposing analyticity upon the reflection coefficients; these poles give rise to explicit solitons [18] beyond the normal simplepole formalism. However, for the spectral equation (1), there still remain questions of the forms and effects of multi-boundary jumps of the continuum spectral data, and of the explicit representations for such jumps and for high-order poles. Apart from answering these questions we shall in this work consider also the effect of the general

\footnotetext{
${ }^{1}$ Department of Mathematics Statistics and Computing Science, University of New England, Armidale NSW 2351, Australia.

(C) Australian Mathematical Society, 1996, Serial-fee code 0334-2700/95
} 
mathematical scattering and the deviation on the calculation of the time evolution of the spectral data, largely due to (and particular to) the Fredholm formalism for the eigenfunction equations. In contrast to the higher-space dimensional cases where the Inverse Spectral Methods (ISMs) are typically a $\bar{\partial}$-problem $[1,3,4,9-11,14,16,17]$, the ISMs in one space dimension are almost always a Riemann-Hilbert problem.

In Section 2, we shall formulate an ISM for (1) with our newly-added solution for multi-boundary jumps and high-order poles for the spectral data, along with the corresponding explicit representations. We then consider in Section 3 the relationship between the inverse data and the scattering data, and the time evolution of spectral data within our new scheme. Examples are given and certain Levitan equations are also derived. We remark that NEEs related to Lax pairs (or solvable via ISMs) are in general good candidates for complete integrability which is even more so in the case of dynamical systems [15].

To conclude this section, we remark that we shall always assume, whenever the need for more rigour arises, that the magnitude of the potential $u(x)$ decreases sufficiently quickly as $|x| \rightarrow \infty$, as is assumed routinely in the literature.

\section{Multi-boundary jumps and high-order poles.}

Let $\psi \equiv \phi(x, k) \exp (\lambda(k) x)$. It is easy to verify that $\psi$ is a solution of (1) if $\phi$ satisfies

$$
\phi(x, k)=e+g(x, k) *\left[u\left(x^{\prime}, k\right) \phi\left(x^{\prime}, k\right)\right], \quad k \in \mathbb{C} .
$$

Here $e \equiv\left(e_{1}, \ldots, e_{N}\right)$ is the $N \times N$ unity matrix, the convolution $*$ is defined element-wise for matrices via

$$
\begin{gathered}
(p * q)(x) \equiv p(x) * q\left(x^{\prime}\right)=\int_{\mathbb{R}} p\left(x-x^{\prime}\right) \circ q\left(x^{\prime}\right) d x^{\prime}, \\
p \circ q=\left(\begin{array}{ccc}
p_{11} q_{11} & \ldots & p_{1 n} q_{1 n} \\
\ldots & \ldots & \cdots \\
p_{m 1} q_{m 1} & \cdots & p_{m n} q_{m n}
\end{array}\right),
\end{gathered}
$$

where $\mathbb{R}$ is the field of real numbers and the matrix Green's function $g=\left(g_{l m}\right)_{N \times N}$ is given by

$$
g_{l m}(x, k ; \eta)=e^{\left(\lambda_{l}(k)-\lambda_{m}(k)\right) x}\left\{\vartheta(x)-\vartheta\left(\operatorname{Re}\left(\lambda_{l}-\lambda_{m}\right)\right)-\eta_{l m} \cdot \rho\left(\operatorname{Re}\left(\lambda_{l}-\lambda_{m}\right)\right)\right\}
$$

in which $\vartheta(x)=1$ if $x>0$ and $=0$ otherwise, $\rho(x)=1$ if $x=0$ and $=0$ otherwise and $\eta_{l m} \in\{0,1\}$ are constants. For notational simplicity, we shall for any matrix $w$ always denote by $w_{i}$ the $i$-th column and by $w_{i j}$ the $(i, j)$-th element. We may now rewrite (3) in a simpler form as

$$
g_{l m} \equiv G_{l m}^{\epsilon}(x, k)=e^{\left(\lambda_{l}(k)-\lambda_{m}(k)\right) x}\left\{\vartheta(x)-\epsilon_{l m}(k) \Theta\left(\operatorname{Re}\left(\lambda_{l}-\lambda_{m}\right)\right)\right\},
$$


where $\Theta(0)=1$ and $\Theta(x)=\vartheta(x)$ for $x \neq 0$. The index matrix $\epsilon(k)$ we are interested in is therefore characterised by the following three conditions for $l \neq m$ : (i) $\epsilon_{m m}(k)=1 \forall k \in \mathbb{C}$ or $\epsilon_{m m}(k)=0 \forall k \in \mathbb{C}$; (ii) $\epsilon_{l m}(k)=1$ if $k \notin \gamma_{l m}$; (iii) $\epsilon_{l m}(k)=\eta_{l m}=$ constant $\forall k \in \gamma_{l m}$ (up to any negligible finite points). Such choice of $\epsilon$ in (4) or $\eta$ in (3) reflects the nonuniqueness of the solutions of (1) on a boundary $\gamma$ (see definition below). The solution $\phi$ will be denoted by $\phi^{\epsilon}$.

Let $\gamma=\cup_{m=1}^{N} \gamma_{m}, \gamma_{m}=\cup_{l=1}^{N} \gamma_{l m}, \gamma_{l l}=\emptyset$ (empty set) and

$$
\gamma_{l m}=\left\{k \in \mathbb{C}: \operatorname{Re}\left(\lambda_{l}(k)-\lambda_{m}(k)\right)=0\right\}, \quad l \neq m .
$$

We define the two sides $\gamma^{ \pm}$of the boundary $\gamma$ such that they enclose any bounded region in $\mathbb{C}$ along with a sufficiently large anti-clockwise circle. On the boundary $\gamma$, we are particularly interested in the three matrix eigenfunctions $\phi$ and $\phi^{ \pm}$determined by (cf (2))

$$
\phi=e+G *(u \phi), \quad \phi^{ \pm}=e+G^{ \pm} *\left(u \phi^{ \pm}\right),
$$

where $G_{l m}^{ \pm}=\lim _{z^{\prime} \rightarrow z \in \gamma^{ \pm}} G\left(x, z^{\prime}\right)$ (that is, limiting values at the sides) and

$$
G_{l m}(x, z)=\exp \left\{\left(\lambda_{l}(k)-\lambda_{m}(k)\right) x\right\}\left\{\vartheta(x)-\vartheta\left(\operatorname{Re}\left(\lambda_{l}(k)-\lambda_{m}(k)\right)\right)\right\} .
$$

These eigenfunctions can be cast into the form of (2) with (4) because $\phi=\phi^{\epsilon^{0}}$ and $\phi^{ \pm}=\phi^{\epsilon^{ \pm}}$if $\epsilon_{m m}^{0} \equiv \epsilon_{m m}^{ \pm} \equiv 0$ and for $l \neq m$

$$
\begin{aligned}
& \epsilon_{l m}^{0}(k)= \begin{cases}0 & k \in \gamma_{l m} \\
1 & \text { otherwise }\end{cases} \\
& \epsilon_{l m}^{ \pm}(k)= \begin{cases}\lim _{z^{\prime} \rightarrow z \in \gamma^{ \pm}} \vartheta\left(\operatorname{Re}\left(\lambda_{l}(k)-\lambda_{m}(k)\right)\right) & k \in \gamma_{l m} \\
1 & \text { otherwise. }\end{cases}
\end{aligned}
$$

For any index matrix $\epsilon$, we can show for $k \in \gamma_{m}$ that

$$
\begin{aligned}
\phi_{m}^{\epsilon} & (x, k)-\phi_{m}(x, k) \\
& =\sum_{l=1}^{N}\left(\epsilon_{l m}^{0}-\epsilon_{l m}\right) R_{l m}^{\epsilon} \exp \left\{\left(\lambda_{l}-\lambda_{m}\right) x\right\} e_{l}+G_{m} *\left[u\left(\phi_{m}^{\epsilon}-\phi_{m}\right)\right] \\
& =\sum_{l=1}^{N}\left(\epsilon_{l m}^{0}-\epsilon_{l m}\right) R_{l m}^{\epsilon} \phi_{l} \exp \left\{\left(\lambda_{l}-\lambda_{m}\right) x\right\} \\
& =\sum_{l=1, \gamma_{l} \neq \emptyset}^{N}-\epsilon_{l m}(k) R_{l m}^{\epsilon}(k) \phi_{l}(x, k) \exp \left\{\left(\lambda_{l}(k)-\lambda_{m}(k)\right) x\right\}
\end{aligned}
$$


(that is, $\psi^{\epsilon}=\psi\left(e+R^{\epsilon} \circ\left(\epsilon^{0}-\epsilon\right)\right)$ ), where

$$
R_{l m}^{\epsilon}(k)=\int_{\mathbb{R}} e^{\left(\lambda_{m}(k)-\lambda_{l}(k)\right) x} \cdot\left[u(x, k) \phi^{\epsilon}(x, k)\right]_{l m} d x
$$

and $[\cdot]_{l m}$ denotes the $(l, m)$-th element of the matrix. If we take $\epsilon=\epsilon^{ \pm}$and $\delta_{l m}$ to be the Kronecker symbol, then (7) gives

$$
\begin{aligned}
\phi^{ \pm}(x, k) & =\phi(x, k) e^{\lambda x} T^{ \pm}(k) e^{-\lambda x}, \\
T_{l m}^{ \pm}(k) & = \begin{cases}\delta_{l m}-\epsilon_{l m}^{ \pm}(k) R_{l m}^{ \pm}(k), & k \in \gamma_{l m} \\
\delta_{l m} & \text { otherwise, }\end{cases}
\end{aligned}
$$

where $R^{ \pm} \equiv R^{\epsilon^{ \pm}}$. Suppose we order $\lambda_{m}(k)$ near $\gamma$ by

$$
\begin{array}{ll}
\operatorname{Re} \lambda_{i_{1}}>\cdots>\operatorname{Re} \lambda_{i_{N}} & \left(k \text { near } \gamma^{+}\right), \\
\operatorname{Re} \lambda_{j_{1}}>\cdots>\operatorname{Re} \lambda_{j_{N}} & \left(k \text { near } \gamma^{-}\right)
\end{array}
$$

and define $P_{\tau}$ for any permutation $\tau$ by

$$
\begin{aligned}
{\left[P_{\tau}\right]_{i j} } & =\left\{\begin{array}{ll}
1 & \text { if }(i, j) \in\left\{\left(1, m_{1}\right), \ldots,\left(N, m_{n}\right)\right\} \\
0 & \text { otherwise }
\end{array},\right. \\
\tau & =\left(\begin{array}{ccc}
1 & \ldots & N \\
m_{1} & \ldots & m_{N}
\end{array}\right)
\end{aligned}
$$

and $P^{ \pm}(\varphi)=P_{\tau^{ \pm}} \varphi\left(P_{\tau^{ \pm}}\right)^{-1}$ with $\tau^{+}=\left(\begin{array}{ccc}1 & \ldots & N \\ i_{1} & \ldots & i_{N}\end{array}\right)$ and $\tau^{-}=\left(\begin{array}{ccc}1 & \ldots & N \\ j_{1} & \ldots & j_{N}\end{array}\right)$. If we regard $\phi$ as a matrix representation of a linear operator in $\mathbb{C}^{N}$, then $P^{ \pm}(\phi)$ are the new representations over the same basis set but of different orders. In terms of the new coordinates, relation (9) becomes

$$
P^{ \pm}\left(\phi^{ \pm}(x, k)\right)=P^{ \pm}(\phi(x, k)) e^{P^{ \pm}(\lambda) x} \cdot P^{ \pm}\left(T^{ \pm}(k)\right) \times e^{-P^{ \pm}(\lambda) x},
$$

in which $P^{ \pm}\left(T^{ \pm}(k)\right)$ are, due to (10), either upper- or lower- triangular matrices with all diagonal elements equal to 1 . Hence $\operatorname{det}\left(T^{ \pm}\right)=1$ and (9) gives

$$
\phi^{+}(x, k)=\phi^{-}(x, k) e^{\lambda(k) x} S(k) e^{-\lambda(k) x}, \quad S(k)=\left(\operatorname{adj} T^{-}(k)\right) T^{+}(k), \quad k \in \gamma(11)
$$

If the boundary $\gamma$ is simple in the sense that no proportion of $\gamma_{l m}$ falls into $\gamma_{l^{\prime} m}$ for another $l^{\prime}$, then $\phi$ itself as a base on $\gamma$ is simpler than either $\phi^{+}$or $\phi^{-}$and may even contain more Volterra-type integral equations. Notice that, in contrast to the case of (nonsimple) multi-boundary, $G_{m}$ will be either $G_{m}^{+}$or $G_{m}^{-}$on the whole of $\gamma_{l m}$. Hence 
we can fix a $g=G^{a}$ for $\phi^{a}$ via (2) such that for $k \in \gamma_{l m}, G_{m}^{a}=G_{m}^{ \pm}$if $G_{m}=G_{m}^{\mp}$ and $G^{a}=G$ for other $k \in \mathbb{C}$. Then we have instead of (11)

$$
\phi^{a}(x, k)=\phi(x, k) e^{\lambda(k) x} T(k) e^{-\lambda(k) x}, \quad k \in \gamma,
$$

where $T_{l m}(k)=\delta_{l m} \forall k \notin \gamma_{l m}$, and

$$
T_{l m}(k)=\delta_{l m}-\int_{\mathbb{R}} e^{\left(\left(\lambda_{m}(k)-\lambda_{l}(k)\right) x\right)} \cdot \times\left[u(x, k) \phi^{a}(x, k)\right]_{l m} d x, \quad k \in \gamma_{l m} .
$$

We note that in a true multi-boundary case, one can not simply take $\phi$ alone as a base because $\phi_{m}$ may be neither $\phi_{m}^{+}$nor $\phi_{m}^{-}$on $\gamma_{l m}$, but a measurement of $\phi^{+}-\phi^{-}$is necessary for the final inverse from spectral data. Form (12) is similar to the case of $[7,8]$ but the formalism is simpler.

We now proceed to define the discrete spectral data for high-order poles, stress laying on the explicit representations in terms of the eigenfunctions. Let $\Gamma_{m}$ be the set of poles of $\psi_{m}$ in $\mathbb{C} \backslash \gamma$ (with non-presence of other singularities assumed). We denote by $k_{m ; s}$ the $s$-th pole of $\psi_{m}(k)$ of order $n_{m ; s}$. For simplicity we assume as usual $\Gamma_{m} \cap \Gamma_{m^{\prime}}=\emptyset$ for $m \neq m^{\prime}$ and we shall often denote $k_{m ; s}$ by $k_{\alpha}$ through an index pair $\alpha=(m ; s)$. For any $k_{\alpha} \in \Gamma_{m}$, there exists a $\lambda_{m^{\prime}}\left(m^{\prime} \neq m\right)$ such that $\operatorname{Re} \lambda_{m^{\prime}}>\operatorname{Re} \lambda_{m}$, for otherwise $\phi$ would be of Volterra type equation and thus analytic near $k=k_{\alpha}$. We denote by $\lambda_{\sigma(\alpha)}\left(>\lambda_{m}\left(k_{\alpha}\right)\right)$ the one which attains the smallest value of such $\operatorname{Re} \lambda_{m}$. Then the discrete spectral data $b_{\alpha}^{(l)}$ for $0 \leq l \leq n_{\alpha}-1$, associated with the pole at $k=k_{\alpha}$ of order $n_{\alpha}$ with $\alpha=(m ; s)$, are given by

$$
b_{\alpha}^{(l)}=-\int_{\mathbb{R}} e_{\sigma}^{T}\left(\exp \left\{-\lambda_{\sigma}(k) x\right\} \psi_{m}(x, k) \times\left(k-k_{\alpha}\right)^{n_{\alpha}}\right)^{(l)}{ }_{k=k_{\alpha}} d x, \quad \sigma=\sigma(\alpha) \in\{1, \ldots, N\},
$$

where ${ }^{T}$ denotes matrix or vector transposition. This establishes the important expansion

$$
\psi_{m}(x, k)=\sum_{j=1}^{n_{\alpha}} \varphi_{\alpha}^{\left(n_{\alpha}-j\right)}\left(x, k_{\alpha}\right) /\left(\left(n_{\alpha}-j\right) !\left(k-k_{\alpha}\right)^{j}\right)+\mathscr{O}(1)
$$

in which

$$
\begin{aligned}
\varphi_{\alpha}(x, k) & \equiv \psi_{m}(x, k)\left(k-k_{\alpha}\right)^{n_{\alpha}}, \\
\varphi_{\alpha}^{(l)}\left(x, k_{\alpha}\right) & =\sum_{j=0}^{l}\left(\begin{array}{l}
l \\
j
\end{array}\right) b_{\alpha}^{(j)} \psi_{\sigma(\alpha)}^{(l-j)}\left(x, k_{\alpha}\right) \equiv\left(b_{\alpha} \mid \psi_{\sigma(\alpha)}\right)^{(l)},
\end{aligned}
$$

where $\left(\begin{array}{l}l \\ j\end{array}\right)=l ! /(j !(l-j) !),(l)$ denotes the $l$-th derivative with respect to $k$ and the algebraic operator $\langle l\rangle$ is defined by

$$
\left(h_{1}|\ldots| h_{m}\right)^{\langle l\rangle}=\sum_{j_{1}+\ldots+j_{m}=l}^{0 \leq j_{i} \leq l}\left(\begin{array}{c}
l \\
j_{1} \ldots j_{m}
\end{array}\right) h_{1}^{\left\langle j_{1}\right\rangle} \ldots h_{m}^{\left\langle j_{m}\right\rangle}, \quad\left(\begin{array}{c}
l \\
j_{1} \ldots j_{m}
\end{array}\right) \equiv \frac{l !}{j_{1} ! \ldots j_{m} !} .
$$


We note that the above operator $\langle l\rangle$ plays a somewhat algebraic role for the differential operator $\partial_{k}^{l}$ and satisfies $(f \mid(g+h))^{(l)}=(f \mid g)^{(l)}+(f \mid h)^{(l)}$ whenever $(g+h)^{(j)}=$ $g^{(j)}+h^{\langle j\rangle}$ for $0 \leq j \leq l$, and $(f|g| h)^{\langle l\rangle}=((f \mid g) \mid h)^{\langle l\rangle}=(f \mid(g \mid h))^{\langle l\rangle}$. This operator is used here because the analyticity of $b^{(l)}$ is not assumed. We now prove (15). First from (5), we have at $k=k_{\alpha}$ that

$$
\psi_{\sigma}^{(l)}\left(x, k_{\alpha}\right)=F_{\sigma ; l}\left(x, k_{\alpha}\right) e^{\lambda_{\sigma} x} \cdot e_{\sigma}+\sum_{j=0}^{l}\left(\begin{array}{l}
l \\
j
\end{array}\right) \times \int_{\mathbb{R}} D_{\sigma}^{(j)}\left(x, x^{\prime}, k_{\alpha}\right) \psi_{\sigma}^{(l-j)}\left(x^{\prime}, k_{\alpha}\right) d x^{\prime},
$$

where

$$
\begin{aligned}
& D_{m}\left(x, x^{\prime}, k\right)=e^{\lambda(k)\left(x-x^{\prime}\right)}\left\{\vartheta\left(x-x^{\prime}\right)\right. \\
&\left.-\operatorname{diag}\left(\vartheta\left(\operatorname{Re}\left(\lambda_{1}-\lambda_{m}\right)\right), \ldots, \vartheta\left(\operatorname{Re}\left(\lambda_{N}-\lambda_{m}\right)\right)\right)\right\} u\left(x^{\prime}, k\right), \\
& F_{\sigma ; l}(x, k)=\exp \left\{-\lambda_{\sigma}(k) x\right\} \partial_{k}^{l} \exp \left\{\lambda_{\sigma}(k) x\right\} .
\end{aligned}
$$

Since $\varphi_{\alpha}$ satisfies the integral equation

$$
\varphi_{\alpha}(x, k)=e^{\lambda_{m}(k) x}\left(k-k_{\alpha}\right)^{n_{\alpha}} \cdot e_{m}+\int_{\mathbb{R}} D_{m}\left(x, x^{\prime}, k\right) \times \varphi_{\alpha}\left(x^{\prime}, k\right) d x^{\prime}
$$

and the kernel $D_{m}\left(x, x^{\prime}, k\right)$ near $k=k_{\alpha}$ can be represented by

$$
D_{m}\left(x, x^{\prime}, k\right)=D_{\sigma}\left(x, x^{\prime}, k\right)-e^{\lambda_{\sigma}(k)\left(x-x^{\prime}\right)} \cdot e_{\sigma} e_{\sigma}^{T} \cdot u\left(x^{\prime}, k\right),
$$

we have for $0 \leq l \leq n_{\alpha}-1$ that

$$
\begin{aligned}
\varphi_{\alpha}^{(l)}\left(x, k_{\alpha}\right)= & \sum_{j=0}^{l}\left(\begin{array}{l}
l \\
j
\end{array}\right) \int_{\mathbb{R}} D_{\sigma}^{(j)}\left(x, x^{\prime}, k_{\alpha}\right) \varphi_{\alpha}^{(l-j)}\left(x^{\prime}, k_{\alpha}\right) d x^{\prime} \\
& -\left.\sum_{j=0}^{l}\left(\begin{array}{l}
l \\
j
\end{array}\right) F_{\sigma ; j}\left(x, k_{\alpha}\right) e^{\lambda_{\sigma} x} \cdot e_{\sigma} e_{\sigma}^{T} \cdot \partial_{k}^{l-j}\right|_{k=k_{\alpha}} \times \int_{\mathbb{R}} e^{-\lambda_{\sigma} x^{\prime}} u\left(x^{\prime}, k\right) \varphi_{\alpha}\left(x^{\prime}, k\right) d x^{\prime} \\
= & \sum_{j=0}^{l}\left(\begin{array}{l}
l \\
j
\end{array}\right)\left\{b^{(l-j)} F_{\sigma ; j}\left(x, k_{\alpha}\right) e^{\lambda_{\sigma} x} \cdot e_{\sigma}\right. \\
& \left.+\int_{\mathbb{R}} D_{\sigma}^{(j)}\left(x, x^{\prime}, k_{\alpha}\right) \varphi_{\alpha}^{(l-j)}\left(x^{\prime}, k_{\alpha}\right) d x^{\prime}\right\} .
\end{aligned}
$$

The solution of (18) is obviously that in (16) due to (17). An alternative way of constructing discrete spectral data without the explicit expression (14) and (15) is to prove inductively a more general form

$$
\varphi_{\alpha}^{(l)}\left(x, k_{\alpha}\right)=\sum_{i=1, i \neq m}^{N} \sum_{j=0}^{l}\left(\begin{array}{l}
l \\
j
\end{array}\right) b_{\alpha ; i}^{(j)} \cdot \psi_{i}^{(l-j)}\left(x, k_{\alpha}\right)
$$


$\operatorname{via} \Psi(x, k) \equiv \operatorname{det}\left(\varphi_{\alpha}, \psi_{1} \ldots, \psi_{m-1}, \psi_{m+1}, \ldots, \psi_{N}\right)$ and

$$
\begin{aligned}
& \Psi^{(l+1)}\left(x, k_{\alpha}\right) \\
& \left.\equiv \operatorname{det}\left(\varphi_{\alpha}^{(l+1)}-\sum_{i=1, i \neq m}^{N} \sum_{j=0}^{l}(l+1 j) b_{\alpha ; i}^{(j)} \cdot \psi_{i}^{(l+1-j)}, \psi_{1}, \ldots, \psi_{m-1}, \psi_{m+1}, \ldots, \psi_{N}\right)\right|_{k=k_{\alpha}} \\
& \quad+\left.\sum_{i=1, i \neq m}^{N} \sum_{j=0}^{l}\left(\begin{array}{c}
l+1 \\
j
\end{array}\right) b_{\alpha ; i}^{(j)} \cdot \partial_{k}^{l+1-j}\right|_{k=k_{\alpha}} \operatorname{det}\left(\psi_{i}, \psi_{1}, \ldots, \psi_{m-1}, \psi_{m+1}, \ldots, \psi_{N}\right) .
\end{aligned}
$$

This algebraic method, however, tends to induce more discrete inverse data [13] than those given explicitly here in (14) and (15).

To summarise, the complete set of inverse data is given by

$$
\begin{aligned}
\mathscr{S}=\left\{R_{l m}^{ \pm}(\xi), k_{m ; s}, b_{m ; s}^{(j)}: \xi\right. & \in \gamma_{l m} ; l, m=1, \ldots, N, l \neq m \\
s & \left.=1, \ldots, N_{m} ; j=0, \ldots, n_{m ; s}-1\right\} .
\end{aligned}
$$

We note that only half of the $R_{l m}^{ \pm}$are actually needed to construct the jump measurement $S(\xi)$ from (11). Also in the case of a different but simpler procedure for simple boundary jumps (12), a different half set $R_{l m}^{a}$ may be used to construct $T(\xi)$ in (13).

For the inverse, one needs to find the eigenfunction $\phi(x, t, k)$ from the inverse (spectral) data:- the expansion of eigenfunction in terms of $k$, depending upon the specific spectral problems, will then be required to derive potential $\mathrm{u}$. Let $\phi_{m}(x, k)=$ $\sum_{j=1}^{n_{\alpha}} \nu_{\alpha ; j}(x) /\left(\left(n_{\alpha}-j\right) !\left(k-k_{\alpha}\right)^{j}\right)+\mathscr{O}(1)$. Then (15) implies

$$
\begin{aligned}
& \nu_{\alpha ; j}=\left.\partial_{k}^{n_{\alpha}-j}\right|_{k=k_{\alpha}}\left[\varphi_{\alpha}(x, k) e^{-\lambda_{m}(k) x}\right]
\end{aligned}
$$

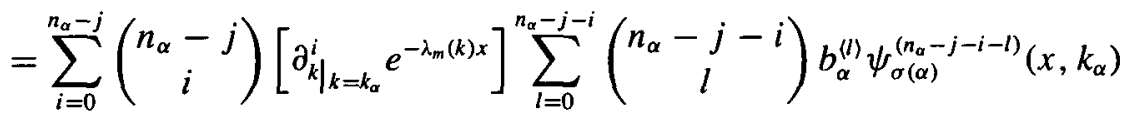

$$
\begin{aligned}
& =\sum_{l=0}^{n_{\alpha}-j}\left(\begin{array}{c}
n_{\alpha}-j \\
l
\end{array}\right) b_{\alpha}^{(l)}\left[\partial_{k}^{n_{\alpha}-j-l} \psi_{\sigma(\alpha)}(x, k) \times e^{-\lambda_{m}(k) x}\right]_{k=k_{\alpha}} \\
& =\left(b_{\alpha}\left|\psi_{\sigma(\alpha)}\left(x, k_{\alpha}\right)\right| e^{-\lambda_{m}\left(k_{\alpha}\right) x}\right)^{\left\langle n_{\alpha}-j\right\rangle},
\end{aligned}
$$

where $\alpha=(m ; k), h^{(l)}\left(k_{\alpha}\right)=\partial_{\left.k\right|_{k=k_{\alpha}}}^{l} h(k)$ is defined for $h=\psi_{\sigma(\alpha)}(x, k)$ and $h=$ $e^{-\lambda_{m}(k) x}$. If we rewrite $(11-13)$ as

$\psi^{+}-\psi^{-}=\psi^{ \pm} Q^{ \pm}, \quad Q^{+}=S-e, Q^{-}=e-S^{-1} ; \quad \psi^{a}-\psi=\psi Q, \quad Q=T-e$

then the integral equations for the eigenfunctions are given (via (20) and the Cauchy 
residue formula) by

$$
\begin{aligned}
& \psi_{m}^{ \pm}(x, k) \\
& =e^{\lambda_{m}(k) x} e_{m}+\frac{1}{2 \pi i} \sum_{l=1, l \neq m}^{N} \int_{\gamma_{m}} \frac{\kappa_{l m}\left(k^{\prime}\right)}{k^{\prime}-k^{ \pm}} Q_{l m}^{\beta}\left(k^{\prime}\right) \psi_{l}^{\beta}\left(x, k^{\prime}\right) e^{\left(\lambda_{m}(k)-\lambda_{m}\left(k^{\prime}\right)\right) x} d k^{\prime} \\
& \quad+\sum_{s=1, \alpha=(m ; s)}^{N_{m}} \sum_{j=1}^{n_{\alpha}} \frac{e^{\lambda_{m}(k) x}}{\left(n_{\alpha}-j\right) !\left(k-k_{\alpha}\right)^{j}}\left(b_{\alpha}\left|\psi_{\sigma(\alpha)}\left(x, k_{\alpha}\right)\right| e^{-\lambda_{m}\left(k_{\alpha}\right) x}\right)^{\left(n_{\alpha}-j\right)},(\forall \beta= \pm)
\end{aligned}
$$

(where $\kappa_{l m} \equiv 1$ ) and their derivatives with respect to $\mathrm{k}$. In the case of only simple boundary jumps (and so $R_{m}^{a}=R_{m}^{ \pm}$and $Q_{m}=-R_{m}^{a}$ ), we may solve them from a simpler set of equations which is (21) with $\psi^{ \pm}$replaced by $\psi^{a}, Q^{\beta}$ by $Q, \psi^{\beta}$ by $\psi$, and $\kappa_{l m}(k)=1$ if $G_{m}=G^{-}$for $k \in \gamma_{l m}$ and $=-1$ otherwise.

\section{Scattering and other auxiliaries}

Although the inverse data are essentially the scattering data in the classical ZSAKNS spectral problem [2], inverse data are in general not scattering data. This is particularly true in many dimensions [9]. First let us link up (7) and (8) by the relation

$$
\begin{aligned}
R^{\epsilon}(k) & =\int_{\mathbb{R}} e^{-\lambda x} u \phi^{\epsilon} e^{\lambda x} d x \\
& =\int_{\mathbb{R}} e^{-\lambda x} u \psi \cdot\left(e+R^{\epsilon} \circ\left(\epsilon^{0}-\epsilon\right)\right) d x \\
& =R(k)\left(e+R^{\epsilon} \circ\left(\epsilon^{0}-\epsilon\right)\right) .
\end{aligned}
$$

From (2) and (4), the space asymptotic (scattering) gives us

$$
\psi^{\epsilon}(x, k) \approx e^{\lambda(k) x} S_{ \pm}^{\epsilon}(k), \quad \text { as } x \rightarrow \pm \infty
$$

where

$$
S_{ \pm}^{\epsilon}(k)=e+\left(\left[\frac{1 \pm 1}{2}-\epsilon_{l m}(k) \Theta\left(\operatorname{Re}\left(\lambda_{l}-\lambda_{m}\right)\right)\right] R_{l m}^{\epsilon}(k)\right)_{N \times N} .
$$

Hence we can represent our inverse data (9) and (11) via the above scattering data

$$
S(k)=\left(S_{-}^{-}(k)\right)^{-1} S_{-}^{+}(k), \quad \text { or } \quad R^{\epsilon}=S_{+}^{\epsilon}-S_{-}^{\epsilon} .
$$

In fact, we may construct $S$ directly from the elements of $S_{ \pm}^{\epsilon}$ (and so the $R_{l m}^{\epsilon}$ obtained there) so as to avoid the inverse of the matrix $S_{-}^{-}$. Fredholm theory gives the solution for $\phi$ in (5) as

$$
\phi_{m}(x, k)=e_{m}+\frac{1}{f_{m}(k)} \int_{\mathbb{R}} \mathscr{F}_{m}\left(x, x^{\prime}, k\right) e_{m} d x^{\prime},
$$


where $f_{m}$ and $\mathscr{F}_{m}$ are both analytic in $k$ (see for example Appendix of [7] for explicit details) and $f_{m} \rightarrow 1$ and $\phi_{m} \rightarrow e_{m}$ for $|k| \rightarrow \infty$. Therefore we have

$$
\phi_{m m}^{ \pm}(x, k) \approx S_{+m m}^{ \pm}(k)=h_{m}^{ \pm}(k) / f_{m}(k)+1, \quad k \in \gamma_{l m}, x \rightarrow \infty,
$$

and the poles of $\phi$ are determined by zeros of $f_{m}$ in $\mathbb{C} \backslash \gamma$ and hence the poles of $S_{+m m}^{ \pm}$. Thus the scattering data (23) also determine in principle our inverse data (19) as in the classical case $[2,3]$.

As for the time evolution of the spectral data, one requires the time part of a Lax pair $\mathbb{M}=\partial_{t}-\mathbb{A}\left(u, \partial_{x}, k\right)$ which gives NEE via for example $[\mathbb{L}, \mathbb{M}]=0$. One of the standard procedures is to find $\Xi^{\epsilon}(k, t)$ such that $\mathbb{M}\left(\psi^{\epsilon} \Xi^{\epsilon}\right)=0$ (for $\epsilon= \pm$ in particular) as $x \rightarrow \infty$ or $x \rightarrow-\infty$, and then, by applying $\mathbb{M}$ to (11) and (15), for instance $\psi^{+} \Xi^{+}=\left(\psi^{-} \Xi^{-}\right) \cdot\left(\left(\Xi^{-}\right)^{-1} S \Xi^{+}\right)$, one gets the time evolution

$$
\partial_{t}\left(\left(\Xi^{-}(k, t)\right)^{-1} S(k, t) \Xi^{+}(k, t)\right)=0 .
$$

However, it is not obvious in general what such $\Xi^{\epsilon}(k, t)$ are unless some of the scattering matrices $S_{ \pm}^{\epsilon}$ are very simple, such as $S_{+}^{\epsilon}$ or $S_{-}^{\epsilon}=e$ which are exactly the case in ZS-AKNS scheme. Therefore we shall instead give here a more universal procedure. Let $\Omega(k)=\mathbb{A}(u, \lambda(k), k)_{\left.\right|_{u=0}}$. Then as $x \rightarrow \pm \infty$ we have $\left(\partial_{t}-\Omega(k)\right)\left(S_{ \pm}^{\epsilon} \Xi^{\epsilon}\right)=0$, that is,

$$
S_{ \pm}^{\epsilon}(k, t) \Xi^{\epsilon}(k, t)=e^{\Omega(k) t} S_{ \pm}^{\epsilon}(k, 0) \Xi^{\epsilon}(k, 0),
$$

and hence the $\Xi^{\epsilon}$-free relation

$$
S_{+}^{\epsilon}(k, t)\left(S_{-}^{\epsilon}(k, t)\right)^{-1}=e^{\Omega(k) t}\left[S_{+}^{\epsilon}(k, 0) \times\left(S_{-}^{\epsilon}(k, 0)\right)^{-1}\right] e^{-\Omega(k) t}
$$

for the 'reflection coefficients'. These are however not yet of the form of our inverse data. Later we shall show that if the eigenfunctions of the ZS-AKNS spectral problem are given by Fredholm integral equations, we have to use the above procedure (including (22)) to obtain the time evolution because none of $S_{ \pm}^{ \pm}=e$. This seems to be the tradeoff for the neat Fredholm formalism. A similar situation is present also for the ISMs related to the DS-I equations, for which one can either have neater time evolution with a penalty of a more involved inverse scattering transform (IST) [5] or a simpler IST with a more complicated time evolution [10].

As an example, we consider the spectral equation

$$
\psi_{x}=i k \cdot \operatorname{diag}\left(\omega_{1}, \ldots, \omega_{N}\right) \psi+u(x) \psi
$$

where $(N \geq 2), \omega_{i} \in \mathbb{R}, u_{i i}=0$, and without loss of generality $\omega_{1}<0, \omega_{1}<$ $\omega_{2}<\ldots<\omega_{N}, \omega_{m} \neq 0 \forall m$. Its IST has been studied in detail [3, 19] in terms 
of pure mathematical language. However, our purpose here is to give explicitly the eigenfunctions and the related scattering data. It thus provides means for the calculation of the general time evolution. We will, furthermore, give the related Levitan equations.

The index matrices of (6) are defined by $\epsilon^{ \pm}=\epsilon^{0}=1 \forall k \in \mathbb{C} \backslash \mathbb{R}$ and for $k \in \gamma=\mathbb{R}$,

$$
\epsilon^{+}=\left(\begin{array}{cccccc}
0 & 1 & . & . & . & 1 \\
& 0 & 1 & & . & \\
& & & & 0 & 1 \\
& & & & & 0
\end{array}\right), \epsilon^{-}=\left(\begin{array}{lllll}
0 & & & & \\
1 & 0 & & & \\
& & . & & \\
1 & 1 & . & .1 & 0
\end{array}\right), \epsilon^{0}=\left(\begin{array}{llll}
0 & & & \\
& 0 & & \\
& & \cdot & \\
& & 0
\end{array}\right)
$$

Hence from the kernel form $G^{ \pm}$we have via (27) that all $\psi^{ \pm}$as solutions of (5) are meromorphic in $\mathbb{C}^{ \pm}$, with $\psi_{1}^{+}$and $\psi_{N}^{-}$being holomorphic there due to their Volterratype integral kemels.

In order to cast the integral equations (21) into Levitan equations, we rewrite $\psi^{ \pm}$ via the Fourier transform (notice that $\lambda_{m}(k)=i \omega_{m} k$ )

$$
\psi_{m}^{ \pm}(x, k)=e^{\lambda_{m}(k) x} e_{m}+\int_{\mathbb{R}} K_{m}^{ \pm}\left(x, x^{\prime}\right) e^{\lambda_{m}(k) x^{\prime}} d x^{\prime}, \quad k \in \mathbb{R}
$$

In fact, (5), (27) and (28) imply $K_{1}^{+}\left(x, x^{\prime}\right)=K_{N}^{-}\left(x, x^{\prime}\right)=0$ for $x<x^{\prime}$. Let us recall some useful formulas below for $a, x \in \mathbb{R} \backslash\{0\}$.

$$
\begin{aligned}
& \int_{\mathbb{R}} \exp \left\{i a k\left(x-x^{\prime}\right)\right\} d k=2 \pi \delta\left(x-x^{\prime}\right) /|a|, \\
& \int_{\mathbb{R}} \frac{e^{i a k x}}{k^{\prime}-k \mp i 0} d k= \pm 2 \pi i \vartheta(\mp a x) e^{i a k^{\prime} x}, \quad k^{\prime} \in \mathbb{R}, \\
& \int_{\mathbb{R}} \frac{e^{i a k x}}{\left(k-k_{\alpha}\right)^{j}} d k=\frac{2 \pi i(i a x)^{j-1}}{(j-1) !} e^{i a k_{\alpha} x} \times\left(\vartheta\left(k_{\alpha, l}\right)-\vartheta(-a x)\right), \quad j \geq 1, k_{\alpha} \in \mathbb{C} \backslash \mathbb{R} .
\end{aligned}
$$

Multiplying (21) by $\frac{1}{2 \pi} \int_{\mathbb{R}} d k \cdot e^{-i \omega_{m} k x^{\prime}}$, we have for $x \neq x^{\prime}$ and $\beta= \pm$ that

$$
\begin{aligned}
& \frac{1}{\left|\omega_{m}\right|} K_{m}^{ \pm}\left(x, x^{\prime}\right) \\
& =\sum_{l=1, l \neq m}^{N} \pm \vartheta\left(\mp \omega_{m}\left(x-x^{\prime}\right)\right) \frac{1}{2 \pi} \int_{\mathbb{R}} Q_{l m}^{\beta}\left(k^{\prime}\right) e^{-i \omega_{m} k^{\prime} x^{\prime}} \psi_{l}^{\beta}\left(x, k^{\prime}\right) d k^{\prime} \\
& \quad+\sum_{s=1, \alpha=(m ; s)}^{N_{m}}\left(\vartheta\left(k_{\alpha ; l}\right)-\vartheta\left(-\omega_{m}\left(x-x^{\prime}\right)\right)\right) \frac{i}{\left(n_{\alpha}-1\right) !}\left(b_{\alpha}\left|e^{-i \omega_{m} k_{\alpha} x^{\prime}}\right| \psi_{\sigma(\alpha)}\left(x, k_{\alpha}\right)\right)^{\left(n_{\alpha}-1\right)}
\end{aligned}
$$


Hence we have the Levitan equations

$$
\begin{aligned}
K_{m}^{ \pm}\left(x, x^{\prime}\right)= & \pm \sum_{l=1, l \neq m}^{N} \vartheta\left(\mp \omega_{m}\left(x-x^{\prime}\right)\right)\left\{e_{l} \cdot F_{l m}^{\beta}\left(x, x^{\prime}\right)+\int_{\mathbb{R}} K_{l}^{\beta}\left(x, x^{\prime \prime}\right) F_{l m}^{\beta}\left(x^{\prime \prime}, x^{\prime}\right) d x^{\prime \prime}\right\} \\
+ & \sum_{s=1, \alpha=(m, s)}^{N_{m}}\left(\vartheta\left(k_{\alpha, l}\right)-\vartheta\left(-\omega_{m}\left(x-x^{\prime}\right)\right)\right) \\
& \times\left\{e_{\sigma(\alpha)} \cdot F_{\alpha}\left(x, x^{\prime}\right)+\int_{\mathbb{R}} K_{\sigma(\alpha)}^{\delta}\left(x, x^{\prime \prime}\right) F_{\alpha}\left(x^{\prime \prime}, x^{\prime}\right) d x^{\prime \prime}\right\},
\end{aligned}
$$

where $\delta= \pm$ if $k_{\alpha} \in \mathbb{C}^{ \pm}$and

$$
\begin{aligned}
& F_{\alpha}\left(x, x^{\prime}\right)=\frac{i\left|\omega_{m}\right|}{\left(n_{\alpha}-1\right) !}\left(b_{\alpha} \mid e^{\lambda_{\sigma(\alpha)}\left(k_{\alpha}\right) x-\lambda_{m}\left(k_{\alpha}\right) x^{\prime}}\right)^{\left\{n_{\alpha}-1\right\}}, \\
& F_{l m}^{ \pm}\left(x, x^{\prime}\right)=\frac{\left|\omega_{m}\right|}{2 \pi} \int_{\mathbb{R}} Q_{l m}^{ \pm}(k) e^{\lambda_{l}(k) x-\lambda_{m}(k) x^{\prime}} d k .
\end{aligned}
$$

We note that (29) should be regarded as two sets of equations, for $x \geq x^{\prime}$ and for $x \leq x^{\prime}$.

As for the time evolution, we have from (22) and (27) that

$$
R_{m+1}^{+}=-\left(R_{1}, \ldots, R_{m}, 0, \ldots, 0\right) \cdot R_{m+1}^{+}+R \cdot e_{m+1} .
$$

Equation (27) implies that only the strict upper and lower half of $R^{+}$and $R^{-}$respectively are required for the completion of our continuum spectral data $S(k)$. Hence we can solve for them from a subset of (30)

$$
\left(\begin{array}{cccc}
1+R_{11} & R_{12} & & R_{1 m} \\
R_{21} & 1+R_{22} & & R_{2 m} \\
\ldots & \ldots & & \\
R_{m 1} & \ldots & \ldots & 1+R_{m m}
\end{array}\right)\left(\begin{array}{c}
R_{1, m+1}^{+} \\
\vdots \\
R_{m, m+1}^{+}
\end{array}\right)=\left(\begin{array}{c}
R_{1, m+1} \\
\vdots \\
R_{m, m+1}
\end{array}\right)
$$

and its counterpart for $R^{-}$. But the time evolution of $R$ is always simple to calculate because $S_{-}^{\epsilon^{0}} \equiv e$, which gives immediate solution of $S_{+}^{\epsilon^{0}}$ and hence $R$ from (24) or (25). Thus the evolution of $S(k, t)$ can be obtained from (31) and its counterpart for $R^{-}$. As for the discrete spectral data $\left(k_{\alpha}, b_{\alpha}^{(l)}\right)$, we can easily derive $\Xi^{\epsilon}(k, t)=\exp (\Omega(k) t)$ for $k$ near $k_{\alpha}$, then multiply it by (15) and finally, by applying $\mathbb{M}$ to (15), obtain the time evolution of the discrete spectral data for high-order poles.

In the ZS-AKNS spectral equation, that is, (26) with $\omega_{2}=-\omega_{1}=1, k_{1, s}=\bar{k}_{s} \in$ $\mathbb{C}^{-}, k_{2, s}=k_{s} \in \mathbb{C}^{+}, \bar{n}_{s}=n_{1, s}, n_{s}=n_{2, s}, b_{1, s}=\bar{b}_{s}$ and $b_{2, s}=b_{s}$, equation (29) 
becomes

$$
\begin{aligned}
& K_{1}^{+}\left(x, x^{\prime}\right)+\left(\begin{array}{l}
0 \\
1
\end{array}\right) \bar{F}\left(x+x^{\prime}\right)+\int_{-\infty}^{x} K_{2}^{-}\left(x, x^{\prime \prime}\right) \bar{F}\left(x^{\prime \prime}+x^{\prime}\right) d x^{\prime \prime}=0 \\
& K_{2}^{-}\left(x, x^{\prime}\right)+\left(\begin{array}{l}
1 \\
0
\end{array}\right) F\left(x+x^{\prime}\right)+\int_{-\infty}^{x} K_{1}^{+}\left(x, x^{\prime \prime}\right) F\left(x^{\prime \prime}+x^{\prime}\right) d x^{\prime \prime}=0
\end{aligned}
$$

and

$$
\begin{aligned}
& \bar{F}(x)=\sum_{s=1}^{\bar{N}} \frac{i}{\left(\bar{n}_{s}-1\right) !}\left(\bar{b}_{s} \mid e^{i \bar{k}_{s} x}\right)^{\left(\bar{n}_{s}-1\right)}-\frac{1}{2 \pi} \int_{\mathbb{R}} R_{21}^{-}(k) e^{i k x} d k, \\
& F(x)=\sum_{s=1}^{N} \frac{-i}{\left(n_{s}-1\right) !}\left(b_{s} \mid e^{-i k_{s} x}\right)^{\left(n_{s}-1\right)}+\frac{1}{2 \pi} \int_{\mathbb{R}} R_{12}^{+}(k) e^{-i k x} d k,
\end{aligned}
$$

and the solution $u \equiv\left(\begin{array}{ll}0 & q \\ r & 0\end{array}\right)$ is given by $q=2 K_{21}^{-}(x, x)$ and $r=2 K_{12}^{+}(x, x)$. We remark that this formalism is different from but is (neater and) equivalent to that in [12].

Notice that the scatterings are given by

$$
\begin{array}{ll}
S_{-}^{+}=\left(\begin{array}{cc}
1 & -R_{12}^{+} \\
0 & 1
\end{array}\right), & S_{+}^{+}=\left(\begin{array}{cc}
1+R_{11}^{+} & 0 \\
R_{21}^{+} & 1+R_{22}^{+}
\end{array}\right), \\
S_{-}^{-}=\left(\begin{array}{cc}
1 & 0 \\
-R_{21}^{-} & 1
\end{array}\right), & S_{+}^{-}=\left(\begin{array}{cc}
1+R_{11}^{-} & R_{12}^{-} \\
0 & 1+R_{22}^{-}
\end{array}\right),
\end{array}
$$

none of which is a unity matrix. Nevertheless (25) with (32) will still enable us to obtain the time evolution $R_{12}^{+}=R_{12}^{+}(k, 0) e^{-2 \xi t}, R_{21}^{-}=R_{21}^{-}(k, 0) e^{2 \xi t}$, where $\Omega(k) \equiv$ $\operatorname{diag}(-\xi, \xi)$. For the discrete spectral data, we use the procedure outlined in the earlier part of this section. The data can be solved recursively from

$$
\begin{aligned}
& \partial_{t} b_{s}^{(m)}=\sum_{l=0}^{m}-2\left(\begin{array}{c}
m \\
l
\end{array}\right) b_{s}^{(l)} \partial_{k_{\alpha}}^{m-l} \xi\left(k_{\alpha}\right), \\
& \partial_{t} \bar{b}_{s}^{(m)}=\sum_{l=0}^{m} 2\left(\begin{array}{c}
m \\
l
\end{array}\right) \bar{b}_{s}^{(l)} \partial_{\bar{k}_{\alpha}}^{m-l} \xi\left(\bar{k}_{\alpha}\right) .
\end{aligned}
$$

This way the explicit soliton solutions of the related nonlinear evolutions equations such as the NLS equation $i \partial_{\imath} q+\partial_{x}^{2} q+2 q^{*} q^{2}=0$ can be obtained algebraically [12].

\section{Conclusion}

We have analysed the multi-boundary jumps and high-order poles in the spectral data which are a phenomenon specific to the eigenfunctions solved from general Fredholmtype integral equations. Such formalism makes it tractable to represent all the spectral 
data in terms of eigenfunctions. However, multi-boundary jumps can exist in the simplest spectral equations and solutions due to high-order poles are observed by, for example, the Hirota method [18]; they all justify our newly-added efforts. Other features such as the mathematically-oriented scatterings, time evolution and Levitan equations are considered concisely for our specific frame.

\section{Acknowledgement}

The author would like to thank Dr. N. P. Pouliezos for some helpful discussions when the author was in UMIST, Manchester, UK.

\section{References}

[1] M. J. Ablowitz, D. Bar Yaacov and A. S. Fokas, "On the inverse scattering transform of the Kadomtsev-Petiashvili equation", Stud. Appl. Math. 69 (1983) 135-142.

[2] M. J. Ablowitz and H. Segur, Solitons and the inverse scattering transform (SIAM, Philadelphia 1981).

[3] R. Beals and R. R. Coifman, "Inverse scattering and evolution equations", Comm. Pure Appl. Math. 38 (1985) 29-42.

[4] R. Beals and R. R. Coifman, "Scattering and inverse scattering for first order systems. II", Inv. Prob. 3 (1987) 577-593.

[5] M. Boiti, J. J. Leon and F. Pempinelli, "A new spectral transform for the Davey-Stewartson $I$ equation", Phys. Lett. A 141 (1989) 101-107.

[6] R. K. Bullough and P. J. Caudrey (eds.), Solitons, (Springer, Berlin, 1980).

[7] P. J. Caudrey, "The inverse problem for a general $N \times N$ spectral equation", D. Physica 6 (1982) $51-66$.

[8] P. J. Caudrey, in: Soliton theory: a survey of results (A. P. Fordy ed.) (Manchester Univ. Press, 1990).

[9] A. S. Fokas and M. J. Ablowitz, "On the inverse scattering of the time-dependent Schrödinger equation and the associated Kadomtsev-Petviashvili (I) equation", Stud. Appl. Math. 69, (1983) 211-228.

[10] A. S. Fokas and P. M. Santini, "Dromions and a boundary value problem for the the Davey Stewartson I equation", Physica D 44 (1990) 99-130.

[11] Z. Jiang, "Construction of scattering data for a class of multidimensional scattering operators", Inv. Prob. 5 (1989) 349-374; "Non-isospectral problems related to DS and other $2+1$ dimensional nonlinear evolution equations", Inv. Prob. 9 (1993) L1-8; "A matrix spectral problem in one complex space dimension", J. Phys. A 26 (1993) L375-378.

[12] Z. Jiang, "The ZK-ZNKS inverse scattering transform with poles of higher orders", Phys. Lett. A 148 (1990) $57-62$.

[13] Jiang $\mathrm{Z}$, "The $N \times N$ inverse spectral problem with poles of multiple orders", Physica Scripta 45 (1992) 65-68.

[14] Z. Jiang and R. K. Bullough, "Combined $\bar{\partial}$ and Riemann-Hilbert inverse methods for integrable nonlinear evolution equations in $2+1$ dimensions", J. Phys. A 20 (1987) L429. 
[15] Z. Jiang and S. Rauch-Wojciechowski, "Integrable dymamical systems with quadratic and cubic nonlinearities related to graded Lie algebras", J. Math. Phys. 32 (1991) 1720-1732.

[16] J. Leon, "Nonlinear evolutions, spectral transform and solitons in $3+1$ dimensions', Phys. Lett. A 156 (1991) 277-285.

[17] P. C. Sabatier, "Spectral transform for nonlinear evolutions in $N$ dimensional space", Preprint PM/91-11, Universite Montpellier II, Montpellier Cedex 05, France.

[18] M. Takahashi and K. Konno, " $N$ double pole solution for the modified Korteweg-de Vries equation by the Hirota's method", J. Phys. Soc. Japan 58 (1989) 3505-3508.

[19] X. Zhou, "Direct and inverse scattering transforms with arbitrary spectral singularities", Commun. Pure Appl. Math. 42 (1989) 895-938. 